\title{
ANALYSIS OF FOXP3 GENE AND PROTEIN EXPRESSIONS IN RENAL ALLOGRAFT BIOPSIES AND THEIR ASSOCIATION WITH GRAFT OUTCOMES
}

Melina Silva de Loreto, Claus Dieter Dummer, Virna Nowotny Carpio, Gabriel Joelsons, Roberto Ceratti Manfro, Luiz Felipe Santos Gonçalves, Francisco José Verissimo Veronese

Background. The transcription factor FOXP3 is increased in acute rejection in renal transplant recipients, but its influence on graft outcomes and its relation to dendritic cells (DCs) is not yet clear. The aim of this study was to correlate FOXP3 expression with graft outcomes and with DCs in renal graft biopsies. Methods. We assessed 96 kidney transplant recipients undergoing allograft biopsy for cause. FOXP3 mRNA was analyzed by real-time PCR, and FOXP3 protein and DCsCD83+ by immunohistochemistry. The magnitude of FOXP3 expression was established by the receiver operating characteristics curve. Graft function and survival were assessed at 5 years posttransplantation, as well as independent predictors of graft loss. Results. Intra-graft FOXP3 gene and protein expression were significantly correlated $(r=0.541, p<0.001)$. Both FOXP3 mRNA and protein were increased in patients with acute rejection (AR). FOXP3-RNAm high ( $\geq 2.36 \log 10 \mathrm{RNAm}$ ) or FOXP3-protein high ( $\geq 2.5$ FOXP3 + cells/mm2) did not correlate with clinical variables, but patients with FOXP3-RNAm high tended to have higher glomerular filtration rates (GFR) from biopsy to last GFR. Patients with FOXP3-RNAm high had more CD83+ DCs on biopsy, but these cells did not associate to AR. Five-year graft survival was not influenced by either FOXP3 mRNA or protein expressions. Conclusions. FOXP3 mRNA and protein presented a good correlation in archival tissue of renal graft biopsies. Increased FOXP3 expression was found in AR biopsies, and those showing FOXP3RNAm high had more DCs. In this cohort, FOXP3 expression was not associated with better renal graft outcomes. 